\title{
The mechanisms and kinetics of the migration of grain boundaries containing extended defects
}

\author{
C P Race* \\ Max Planck Institut für Eisenforschung, 40237 Düsseldorf, Germany and \\ School of Materials, University of Manchester, Manchester, M13 9PL, United Kingdom \\ R Hadian, J von Pezold, B Grabowski, and J Neugebauer \\ Max Planck Institut für Eisenforschung, 40237 Düsseldorf, Germany
}

\begin{abstract}
A full understanding of the basic processes of grain boundary migration is a fundamental prerequisite for predictive models of microstructural evolution in polycrystalline materials in processing and in service. In a detailed study of the kinetics of a [111] $\Sigma 7$ symmetric tilt boundary, we have previously shown that defect-free, flat grain boundaries, below their roughening temperature, can be strictly immobile in the experimental limit.

Here we present the results of molecular dynamics simulations of grain boundaries containing a variety of "defects". These simulations show that the presence of some of these defects restores the mobility of flat boundaries, even well below the roughening transition temperature. These defects fundamentally alter the mesoscale mechanism of grain boundary migration from one involving homogeneous nucleation to a heterogenous process. At the atomistic level, the crystal lattice reorients via coordinated shuffling of groups of atoms. In the case of flat boundaries, these shuffles must accumulate to form critically stable nuclei, but in the case of boundaries containing defects the shuffling of a small number of atoms at the defects can be sufficient, fundamentally altering the mechanism and kinetics of migration.
\end{abstract}

\section{INTRODUCTION}

The motion of grain boundaries plays a major role in the evolution of the mechanical properties of polycrystalline and nanocrystalline materials. To successfully predict the evolution of the grain structure of such materials during production processes and in application we need understanding of the mechanisms by which grain boundaries migrate. Many theoretical models of microstructural evolution need information about the dynamic properties of grain boundaries, but obtaining such information by experiment is difficult.

One hope is that molecular dynamics (MD) simulations might be used to probe the fundamental kinetic properties of grain boundaries as a function of grain boundary geometry and structure. One approach is to focus on measuring the mobility, $m$, of different grain boundaries [1-13. These mobilities are expected to emerge from an assumed proportionality between the observed velocity, $v$, of a grain boundary and the thermodynamic driving force, $P$, for its motion: $v=m P$ [14. Furthermore, the mobility is assumed to be an intrinsic property of a grain boundary of given geometry - something which is well defined only for a flat grain boundary. However, we have previously shown that atomically flat grain boundaries (i.e. boundaries that are free of structural defects such as steps, disconnections and dislocations when at zero temperature), are generally immobile [15. Such smooth flat boundaries must move via the nucleation and growth of "islands" at the grain boundary

\footnotetext{
* Contact: christopher.race@manchester.ac.uk
}

surface. For such a homogeneous nucleation process the energy barrier increases as the inverse of the driving force, resulting in barrier heights in the order of hundreds of $\mathrm{eV}$ and thus zero mobility in the experimentally-relevant, low-driving-force limit.

In real materials boundaries are rarely, if ever, perfectly flat and defect free. They contain structural features due to boundary curvature or the interaction of extended defects with the boundary and they are finite in extent, meeting other grain boundaries at triple junctions. At higher temperatures, above the roughening transition temperature [16] for a given boundary, the free energy cost of forming defects on the grain boundary becomes zero [15. The presence of defects due to roughness or of structural defects removes the need for a homogeneous nucleation process and thus is likely to determine the mobility of real grain boundaries. As we have noted previously [15], this means that the mobility of a given grain boundary will depend on its defect content and structure and may not be an intrinsic property of the boundary geometry that can be determined by simulating the motion of a flat grain boundary segment.

Here we present the results of MD simulations of grain boundaries containing extended defects, which show how those defects change the mechanisms and kinetics of migration at temperatures below the roughening transition. We find that certain defects relax the need for a homogeneous nucleation process as part of the migration mechanism. These defects render the boundary significantly more mobile and restore the postulated proportionality between the driving force for grain boundary migration and the boundary velocity. 

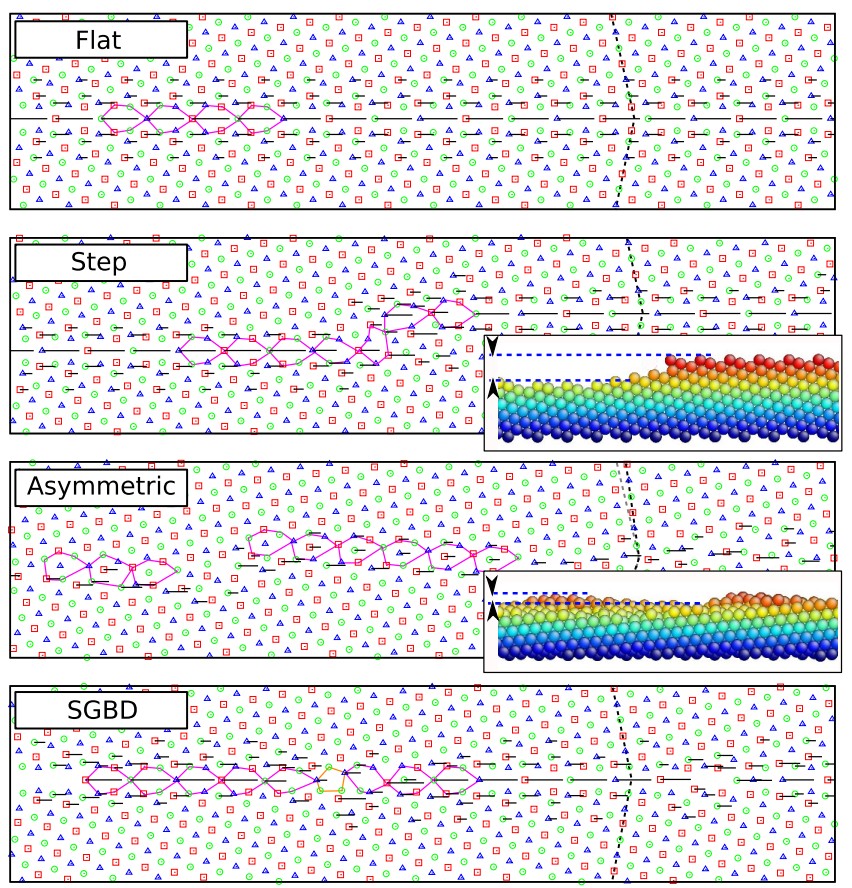

FIG. 1. The unrelaxed of the grain boundary structures used in our simulations. The different symbols denote atoms in different close-packed layers. The solid lines indicate the size of the per atom stress in the direction perpendicular to the grain boundary as a means of visualizing the boundary structure. The polygons in the boundary highlight the basic structural unit of the flat $\Sigma 7$ boundary. The dashed lines indicate $\{1 \overline{1} 0\}$ planes for reference. For the Step and Asymmetric boundaries, the insets show detailed cross-sections through the boundary, with one half of the bicrystal removed and atoms coloured according to their coordinate perpendicular to the boundary, to emphasise the out-of-plane features.

\section{RESULTS AND DISCUSSION}

We have carried out simulations of grain boundaries containing a variety of defects and measured the response of the boundary velocity to variation in temperature and driving force. As a starting point we have taken the [111] $\Sigma 738.21^{\circ}$ symmetric tilt boundary, the kinetics of which we have previously studied in detail [15] and which has been much studied in the literature. We have then introduced three types of "defects" into this boundary, whose structures are shown in Fig. 1. The first and simplest of these is a simple step along the [111] direction with a height of $3.81 \AA$. To make this step "persistent", so that it does not disappear once it has traversed the grain boundary surface, we have applied skewed periodic boundaries to the simulation cell on the plane containing the step direction and the grain boundary normal. The second defect is introduced by adding a slight asymmetry into the boundary by tilting the boundary plane by $4.13^{\circ}$ from the symmetric case. When relaxed, this asymmetric boundary contains large regions that resemble the symmetric boundary (with boundary plane $(12 \overline{3})$ ), separated by small facets on another low index plane (probably $(1 \overline{2} 1)$ or $(1 \overline{1} 0)$, though the plane is uncertain for such small features), as can be seen in Fig. 1. The third defect is introduced by retaining the symmetry of the boundary, but increasing the misorientation angle from $38.21^{\circ}$ to $40.17^{\circ}$. This yields a $\Sigma 9183$ boundary which resembles the $\Sigma 7$ boundary over most of its surface, but contains intrinsic secondary grain boundary dislocations (sgbd), which are required to account for the change in misorientation angle. The three defects above will henceforth be denoted by "Step", "Asymmetric" and "SGBD" respectively, and the original $\Sigma 7$ boundary by "Flat".

The definition of what constitutes a "defect" in the grain boundary surface is, of course, ambiguous. The $\Sigma 7$ boundary itself is not atomically smooth (the (321) plane contains atomic-scale features out of plane) and the Asymmetric and SGBD (symmetric $\Sigma 9183$ ) boundaries are "perfect" CSL (coincident site lattice) [17 boundaries. Here we adopt a definition in which the $\Sigma 7$ boundary is defined as the perfect, defect-free state and our other boundaries are considered as deviations from that state, embodied in the presence of different types of "defects" 18 .

For each of the defective boundaries we carried out a series of grain boundary mobility simulations across a range of temperatures $(490-800 \mathrm{~K})$ and driving forces $(2.5-7.5 \mathrm{meV} /$ atom or approximately $25-75 \mathrm{MPa})$. The inter-atomic forces were modelled using an embedded atom method (EAM) potential [19] and the grain boundary migration was driven using the artificial driving force of Janssens et al. 4. The simulation cell sizes used were: Flat, $420 \times 340 \AA^{2}$, $1160 \mathrm{k}$ atoms; Step, $150 \times 170 \AA^{2}, 210 \mathrm{k}$ atoms; Asymmetric, $210 \times 140 \AA^{2}$, 650k atoms; SGBD, $160 \times 140 \AA^{2}, 730 \mathrm{k}$ atoms. A Nosé-Hoover thermostat was used to maintain a constant temperature and full periodic boundary conditions were applied to the simulation cells. More details of our method can be found in the Appendix and in Refs. 15, 20. In each simulation we measured the average position of the grain boundary across its surface as a function of time and recorded the individual atomic positions every $100 \mathrm{fs}$.

In a first step we compare the behaviour of the defective boundaries with our previous results for a perfect flat boundary segment 15. Fig. 2(a) shows the velocity of the grain boundary as a function of driving force at a temperature of $600 \mathrm{~K}$. In Ref. [15] we reported that the flat $\Sigma 7$ boundary violates the expected proportionality between velocity and driving force and instead shows no discernible motion at low force. We now observe a similar behaviour for the SGBD boundary, although with a higher velocity at any given force. The two other defective boundaries, Step and Asymmetric, show behaviour closer to the classic picture (with non-zero mobility in the zero-force limit). This is particularly striking for the Asymmetric boundary, for which the nominal mobility (we define this as the quotient of the velocity and the driving force, $v / P$ ) can be seen in the inset to Fig. 2(a) 
(a)

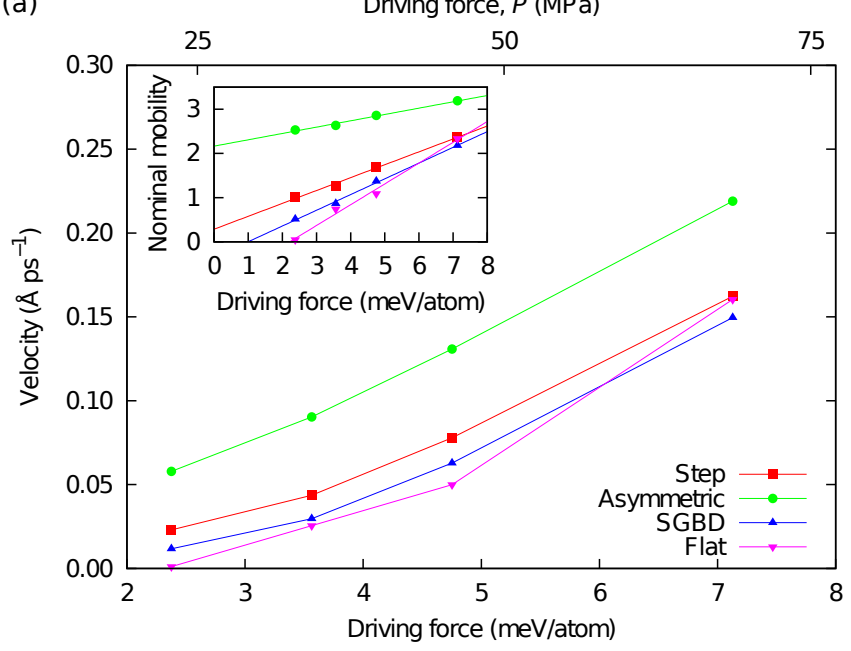

(b)

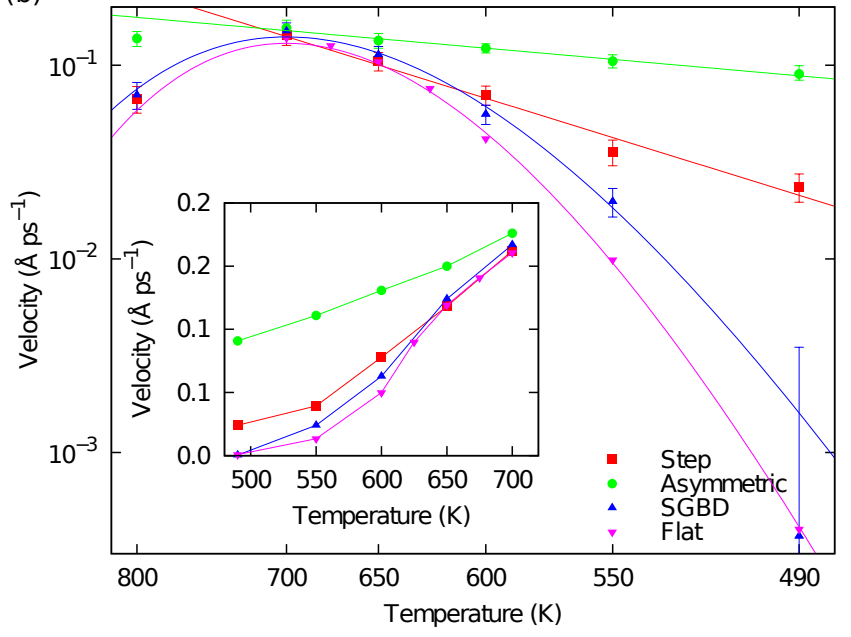

FIG. 2. Mobility data for the grain boundary migration simulations. (a) The grain boundary velocity as a function of driving force at $600 \mathrm{~K}$ for the four different boundaries. The inset shows the same data but presented as a nominal mobility $(\equiv v / P)$ with linear fits to the data. (b) An Arrhenius presentation of the simulation results for a driving force of $5 \mathrm{meV} /$ atom. The solid lines are linear best-fits or fits based on the island nucleation model of migration kinetics. The inset shows the same data on linear scales. Except where indicated, lines are a guide for the eye only.

to be much more weakly dependent on the driving force. This effect of defects in restoring the expected grain boundary mobility has also been observed in simulations of the migration of heterophase solid-solid interfaces [21].

In our previous study [15] we showed that the behaviour of the flat $\Sigma 7$ boundary is a result of the mesoscale mechanism by which the boundary moves. In large, three-dimensional simulation cells the thermodynamically favoured grain grows via the spontaneous formation of "islands" of transformed material at the grain boundary surface. These islands may equivalently be viewed as disconnection loops (in the case of the $\Sigma 7$ boundary the loop is a pure step with no dislocation content). For the grain boundary to migrate, the islands (or disconnection loops) must grow by random fluctuations to become larger than some critical size, beyond which further growth will then be thermodynamically favoured. The migration process at the mesoscale is thus one of homogeneous nucleation involving a competition between the free energy cost of forming the island edge (or disconnection loop) and the stabilising effect of the driving force for grain boundary migration acting over the volume of the island:

$$
F_{\text {island }}(r, P, T)=2 \pi r \phi(T)-\pi r^{2} P d,
$$

where $r$ is the radius of the island, $\phi$ is the excess free energy per unit length of the island edge (or bounding disconnection), $P$ is the driving force for migration (expressed as a pressure) and $d$ is the height of the island [15. Maximising the value of $F_{\text {island }}$ with respect to its radius then gives the free energy of a critical island nucleus:

$$
F_{\mathrm{A}}(P, T)=\frac{\pi[\phi(T)]^{2}}{P d},
$$

This is the free energy barrier for migration of the flat boundary in the experimental limit of low driving force where the migration process is strongly nucleation limited (as opposed to growth limited).

This energy barrier varies with both temperature and driving force. The results for the defective boundaries are compared with those for the Flat boundary in an Arrhenius-like plot in Fig. 2(b) where the temperature dependence of the free energy barrier for the Flat boundary shows up as a strong curvature. As in Ref. [15] we now assume $\phi=\gamma\left(1-T / T_{\mathrm{c}}\right)$, where $\gamma$ is the internal energy per unit length and $T_{\mathrm{c}}$ is the grain boundary roughening temperature. The free energy barrier for homogeneous island nucleation then gives the solid curved lines in Fig. 2(b), which are seen to fit the simulation data very well with independently determined values for $\gamma$ and $T_{\mathrm{c}}$. We see a similar curvature in the results for the SGBD boundary (the line through these data uses a best-fit value for the edge energy $\gamma$ ). For the other two boundaries, however, the results are well fitted by a straight line, which suggests a different mechanism for which there is no variation of the energy barrier with temperature.

To understand the differences between the results for different boundaries we have visualized the shape of the grain boundary surface in each case. The results are shown in Fig. 3. We can see that the Step boundary migrates via motion of the step over the grain boundary surface. The large step height of $3.81 \AA$ is maintained throughout this process. We have also carried out simulations with a step of double this height and found that the larger step quickly dissociates into two smaller steps which repel one another and move separately over the grain boundary surface. In the case of the Asymmetric 


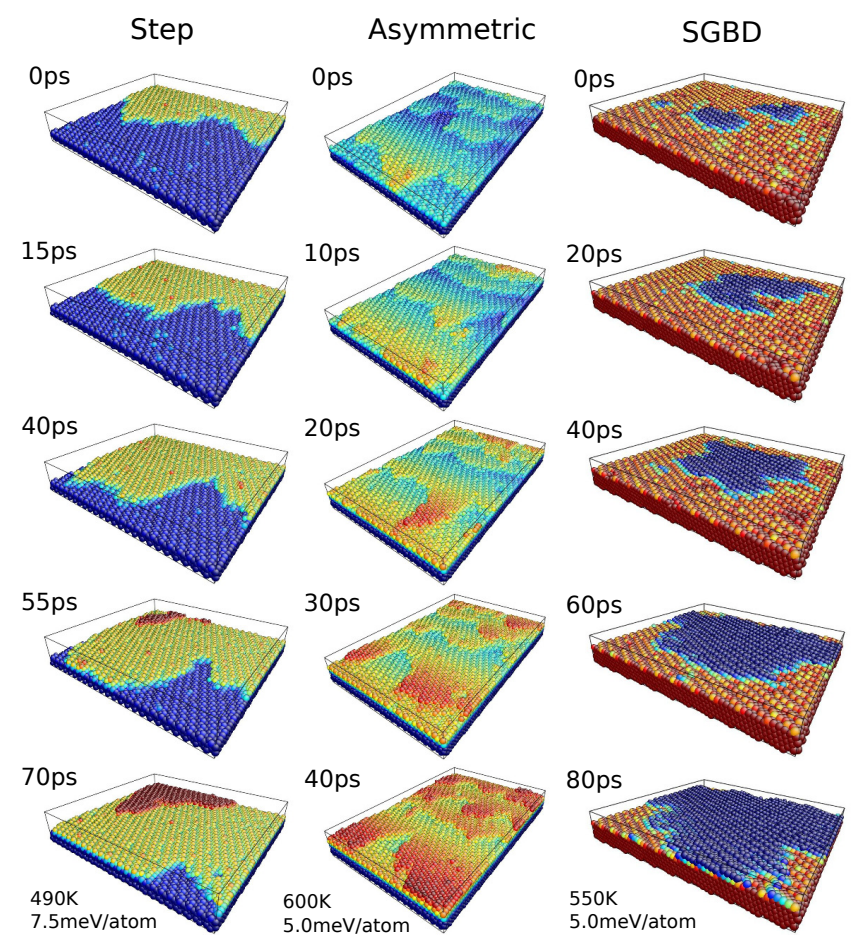

FIG. 3. Snapshots of migrating boundaries containing structural defects. Atoms in one half of the bicrystal are not shown. The atoms are coloured according to their position perpendicular to the grain boundary surface to emphasise the role of out-of-plane features in the migration process (colour scales are arbitrary and vary between defects). Time-scales for the migration process along with the temperatures and driving forces used are marked in the figure.

boundary the out-of-plane features are smaller and we see that the boundary migrates via the motion of these features across the boundary surface. In the case of both the Step and Asymmetric boundaries, migration takes place via processes localized at the out-of-plane features in the defective boundaries. In contrast, we find that the SGBD boundary migrates via the nucleation and growth of islands of transformed material. There are no strong step-like features in this boundary (see Fig. 1) implying that there are no preferential sites for the atomistic shuffling process to take place and so the kinetics are those of island nucleation, as for the Flat boundary.

For the Step and Asymmetric boundaries, the migration mechanism involves the localised rearrangement of the crystal lattice at the step-like features in the boundary surface. In the following we analyse this process in more detail in order to estimate the order of magnitude of the associated energy barrier. The analysis will be performed for the Flat boundary but applies equally well to the other boundaries.

At the atomic level the transformation of crystal from one orientation into the other involves an ordered shuffling of the atoms. This process either takes place at step or facet edges (in the case of the Step and Asymmetric boundaries) or is the mechanism by which the critical island nuclei are built up (in the case of the Flat and SGBD boundaries). The nature of the shuffling process can be seen in the snapshots in Fig. 4 taken from simulations of the migration of the Flat grain boundary. Similar shuffling patterns have been observed both experimentally [22] and in simulations (Ref. 23] presents a thorough analysis of such behaviour). Fig. 4 shows data for the atomic shifts at three different temperatures. At $400 \mathrm{~K}$ the rearrangement of atoms is highly ordered. Some variation from the general pattern occurs even at this low temperature and is highlighted in Fig. 4. The anomalous behaviour takes the form of a sequence of non-standard shift vectors initiated and terminated by hops of atoms between adjacent close-packed layers. Such "strings" of hops have been previously observed and carefully analysed by Zhang and Srolovitz [23. At $600 \mathrm{~K}$ the pattern of rearrangement remains mostly ordered, although some hops between close-packed layers can again be seen. At $800 \mathrm{~K}$ things are dramatically different: the hopping of atoms shows significant disorder and many atoms hop between close-packed layers. This disordering of the process of reorienting the crystal lattice is what causes the velocity of the grain boundary to decrease with increasing temperature above $700 \mathrm{~K}$, the grain boundary roughening temperature (obtained from elevated-temperature simulations of a static grain boundary).

We have used the nudged elastic band (NEB) method 24], as implemented in Lammps, to estimate the energy barrier associated with this shuffling. More details of these calculations are provided in the Appendix. Fig. 5 shows the results of the NEB calculations. We find that to shuffle a rectangular block of 21 atoms (equal to three CSL cells) the barrier is $0.39 \mathrm{eV}$. Shuffling of a 21 atom block maintains the structure of the leading edge of the step in the $\Sigma 7$ boundary. The NEB calculations also reveal that the shuffling of the atoms takes place in three stages with two separate barriers: the nine atoms closest to the boundary are involved in the highest energy step. Six more atoms then hop in a lower energy process, before the final six atoms undergo a barrier-free relaxation. This staged shuffling emerging in the NEB calculations is also evident from a careful analysis of the dynamical simulations and more details are provided in the Appendix. We also note that even a rather large driving force of $25.0 \mathrm{meV}$ atom $^{-1}(\sim 250 \mathrm{MPa})$ does not qualitatively alter the shape of the energy barrier (see inset to Fig. 57). This suggests that large artificial driving forces can be used without affecting the atomistic migration mechanism. However, the active mechanisms at the mesoscale, and so the overall kinetics of migration, can be strongly affected as we have shown previously [15].

Having identified the atomistic process involved in the transformation of crystal volume, we are able to assess the validity of our chosen EAM potential for the purposes of grain boundary simulation. The standard issues of the degree of transferability of empirical potentials mean that, a priori, no assumption of validity should be 
made. We have therefore repeated the NEB barrier calculations using first principles methods as implemented in the plane-wave density functional theory code VASP (see Appendix for details of the calculations and references). We find a value for the energy barrier of $0.31 \mathrm{eV}$. The empirical potential gives a result within $15 \%$ of the first-principles estimate for an equivalent simulation cell.
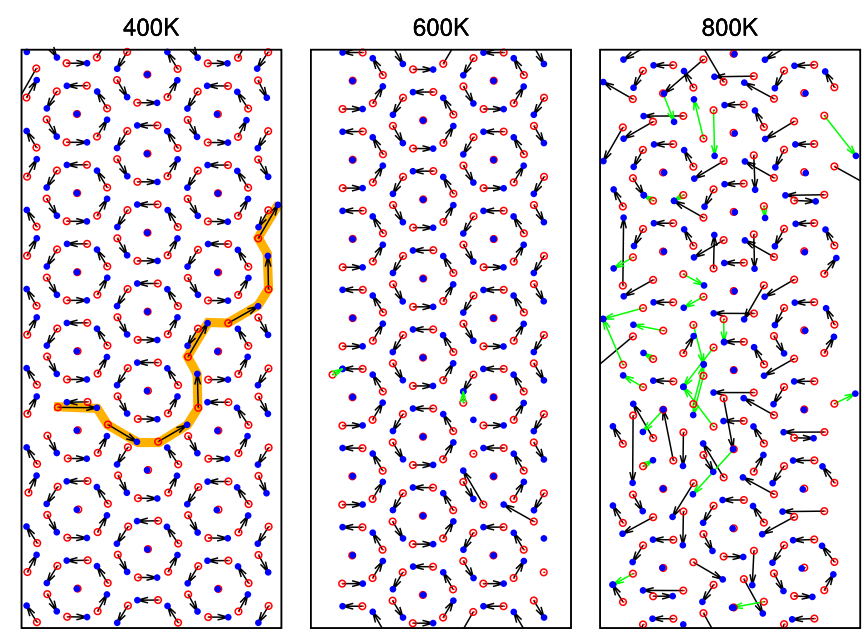

FIG. 4. The patterns of atomic rearrangement in migration simulations at three different temperatures. The initial and final positions are shown by open (red) and filled (blue) symbols respectively. Black arrows show hops confined to a single close-packed plane. Green vectors show atoms hopping between different planes. A "string" 23 of anomalous jumps at $400 \mathrm{~K}$ is highlighted (in orange). In all cases a rigid translation has been applied to the final atom positions to clarify the pattern of rearrangement (see Appendix).

The island-based mechanism of grain boundary migration implies that below $T_{\mathrm{c}}$, boundaries become immobile in the experimental limit [15] because of the inverse relation between driving force and the energy barrier for migration (Eq. 2). This trend can be clearly seen in the data for the Flat and SGBD boundaries in Fig. 6 . in which the activation energy for migration explodes at low force. The activation energies for the Flat and SGBD boundaries are derived from dynamical simulations of grain boundary migration over a range of driving forces and temperatures, by fitting $\gamma$ in Eq. 2 to data for each driving force $\left(T_{\mathrm{c}}\right.$ is fixed at the value derived independently for the Flat boundary). The effect of the strongly increasing driving force should show up as a minimum, "threshold" driving force for migration on a given time-scale. Such thresholds are generally not observed in bicrystal experiments (the work of Kang et al. 25] is an interesting exception). In many cases this is likely to be because the boundaries are studied above their respective roughening transition temperatures. However, given the wide variation of $T_{\mathrm{c}}$ with boundary geometry [16] and the wide range of temperatures studied in the literature, it is also likely that some of the published experimental results are for smooth boundaries, below $T_{\mathrm{c}}$. In this

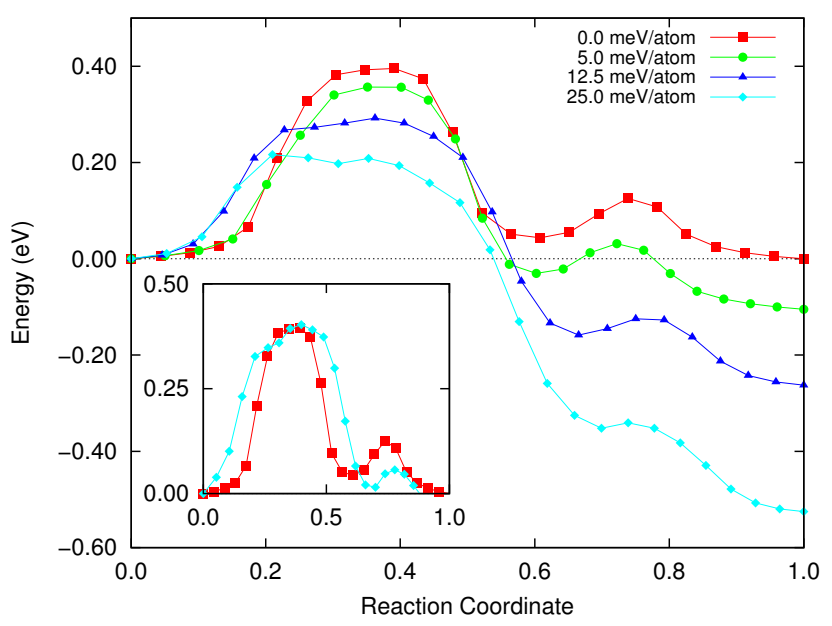

FIG. 5. The energy barriers for atomic rearrangement of 21 atoms derived from NEB calculations over a range of driving forces (each symbol corresponds to a single replica). The inset shows the barriers for no force and the maximum driving force with the energy change due to the artificial driving force subtracted out.

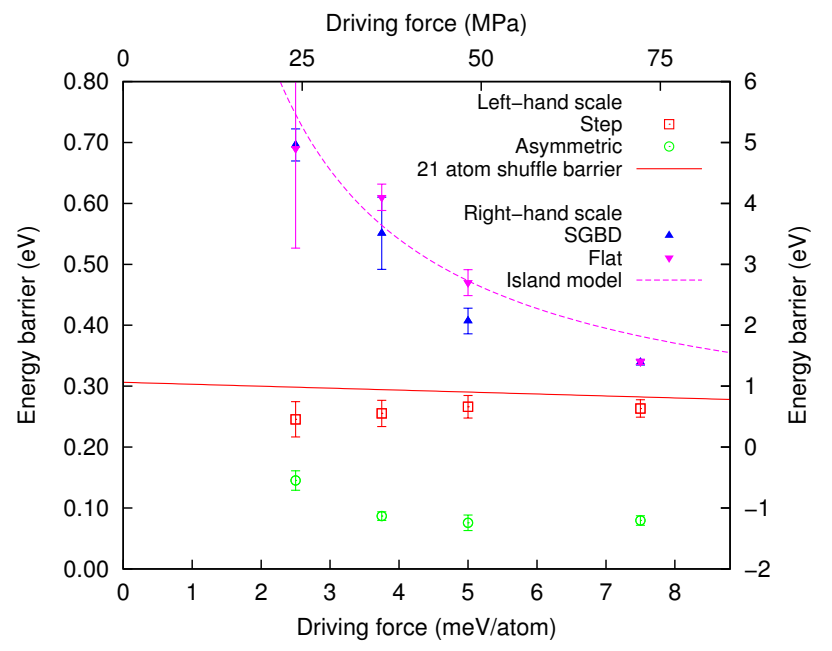

FIG. 6. The energy barriers for grain boundary migration as a function of driving force for the different boundaries. The dashed line is the prediction of the island model of migration kinetics. The solid line shows the barrier for the ordered shuffle of 21 atoms from the disfavoured crystal orientation to the favoured.

case, the observed behaviour, $v \propto P$, could be a result of the presence of defects that relax the need for island nucleation, as shown in our simulation results presented here. Fig 6 emphasises this point: we see no strong upward trend in the activation energy at low driving force for the Step and Asymmetric boundaries.

Fig 6 also shows the energy barrier for the ordered shuffle of twenty-one atoms from the disfavoured orientation to the favoured one [26]. This is comparable to 
the energy barrier for the Step boundary, suggesting that the rate determining step for migration is the shuffling of three CSL units of crystal at the step edge. The barrier for the Asymmetric boundary is somewhat lower and we speculate that the different (smaller) out-of-plane features in this boundary allow it to migrate via the shuffling of smaller numbers of atoms at a time. Though we have not analysed the migration mechanism of these boundaries at the atomistic scale in detail, we have simulated a second asymmetric boundary with a smaller variation of the boundary plane from that in the Flat boundary. This alternative boundary has equivalent out-of-plane features (facets), but at half the spatial frequency of the asymmetric boundary analysed above. As expected, the migration of this boundary is significantly slower. At $500 \mathrm{~K}$ halving the spatial density of the out-of-plane features approximately halves the velocity of the boundary, because each feature has to migrate twice as far across the grain boundary surface in order for the boundary to advance.

A striking feature of Fig. 2(b) is the effect of temperature on the velocities of the different boundaries. As we approach the roughening transition temperature $T_{\mathrm{c}}$ of the $\Sigma 7$ boundary $(\approx 700 \mathrm{~K})$ the velocities for the four different boundaries converge. This behaviour can be explained as follows: above $T_{\mathrm{c}}$ the boundaries will contain a thermal population of out-of-plane features of the same height as the edges of island nuclei and these thermal defects will swamp the effect of the zero-temperature structural defects present in the three defective boundaries. There will be no need for either homogeneous or heterogenous nucleation of atomic shuffles. Above $T_{\mathrm{c}}$ the velocity of the grain boundaries is reduced, due to thermal noise in the atomistic migration mechanism (see Fig. 4(c)). The asymmetric boundary seems to be less prone to this effect meaning, perhaps, that it is somewhat resistant to thermal disordering.

\section{CONCLUSIONS}

In previous work [15] we have shown that low- $\Sigma$ defectfree flat grain boundaries, of the type frequently studied in simulations, can be strictly immobile in the experimental limit. This makes it difficult to define an intrinsic mobility for such boundaries. In the present work we have shown the results of simulations of a variety of grain boundaries that, with reference to a given low- $\Sigma$ boundary, contain defects of various types. Where such defects include significant out-of-plane features in the otherwise flat grain boundary surface, the widely assumed proportionality of migration velocity to driving force is approximately restored. This is due to a change in the mesoscale migration mechanism: in a perfectly flat, defect-free boundary an island-based mechanism, with the kinetics of homogeneous nucleation, prevails, whereas when certain defects are present the migration mechanism is localised at the grain boundary defects. The energy barriers for migration of these boundaries are associated with an atomistic rate-determining step, with a mechanism that depends on the structure of the "defects" in the grain boundary surface. Further detailed study of the mechanisms and kinetics of the migration of grain boundaries containing defects could help to link simulation with experiment and assist in building larger length-scale, physically-informed models of microstructural evolution.

\section{ACKNOWLEDGMENTS}

C.P.R. was funded by a Postdoctoral Fellowship of the Alexander von Humboldt Foundation.

\section{APPENDIX}

\section{A. Molecular dynamics simulations of grain boundary migration}

The grain boundary migration simulations discussed here were carried out using the classical molecular dynamics code Lammps [27. Our simulation cells had periodic boundary conditions in all three dimensions and contained two mutually-cancelling grain boundaries. The inter-atomic forces in our simulations are given by an existing EAM potential for aluminium [19. This potential is fitted to a set of experimental data and the energies of a variety of crystal structures calculated with density functional theory (DFT). The potential functions have an analytical form rather than being piecewise fitted.

Motion of the grain boundaries was driven using the artificial orientation correlated driving force of Janssens et al. 4] implemented in the standard release of Lammps. This force assigns an artificial potential energy to each atom based on the orientation of its local face-centred cubic environment. This artificial potential results in a potential energy penalty for one half of the bicrystal and gives rise to extra artificial forces on atoms close to the grain boundaries. Energy penalties of between 2.5 and $7.5 \mathrm{meV} /$ atom were used.

Because the transformation of crystal volume from the disfavoured to the favoured orientation releases potential energy as heat, we applied a Nose-Hoover thermostat to the entire simulation cell. Temperatures between 400 and $800 \mathrm{~K}$ were simulated. Because grain boundaries give rise to surface-tension-type forces, and because the ratio of grain boundary area to crystal volume is unrealistically high in MD simulations, we chose to fix the dimensions of the simulation cell in the directions parallel to the grain boundary plane. In the perpendicular direction we apply a zero pressure Nose-Hoover barostat to allow volume relaxation. This is required because at elevated temperatures the artificial driving force gives rise to small forces on the atoms in the bulk of the favoured grain that would otherwise cause an excess pressure to build up as the grain boundary migrates. 
The position of the grain boundary is detected by monitoring the profile of the average value of the artificial driving force potential as a function of position normal to the grain boundaries and identifying the position at which it passes through half its maximum. For mapping the grain boundary surface, this analysis is carried out on small portions of the grain boundary surface individually. The velocity of a grain boundary is calculated using the history of its position over time. A measure of the errors in the estimate of velocity is made using bootstrap resampling of the grain boundary trajectory following the method set out in Ref. [20].

\section{B. The atomic shuffling mechanism}

To better understand the variation of the grain boundary velocity with driving force we have mapped the atomic rearrangements necessary to transform crystal volume from the disfavoured to the favoured orientation. Fig. 7 shows plots of the shifts during a simulation of the atom positions in three close-packed layers as the grain boundary sweeps through from left to right, along with histograms of the jump lengths. The atom positions are determined by taking snapshots of the dynamical simulation and relaxing the atoms to their minimum energy configuration. The order inherent in the raw atom shifts becomes more obvious when the final atom positions are adjusted by a rigid translation. This is because the grain boundary has a non-zero excess volume, which shifts the CSL points out of coincidence in a relaxed simulation cell.

\section{The nudged elastic band method}

In Sec. II we presented the results of calculations of the energy barrier for the atomic rearrangements associated with grain boundary migration. These were calculated using the climbing image nudged elastic band (NEB) method [24, 28] implemented in Lammps [27.

In this method, a series of replicas of our system, linearly interpolated between specified initial and final equilibrium configurations, is constructed. Each atom is connected to its representation in the adjacent replicas by a notional "elastic band". The replica systems are then allowed to relax under those components of the EAM interatomic forces acting perpendicular to the bands, plus a force parallel to the bands proportional to the distance between adjacent replicas. The bands thus act to ensure that the replicas remain evenly spaced between the equilibrium configurations.

In a second stage, the forces in the highest energy replica are amended: the band forces are removed and EAM forces parallel to the bands are allowed to act, but with a negative sign. The highest energy replica thus climbs in energy along the bands (i.e. along the minimum energy path) to find the barrier configuration.
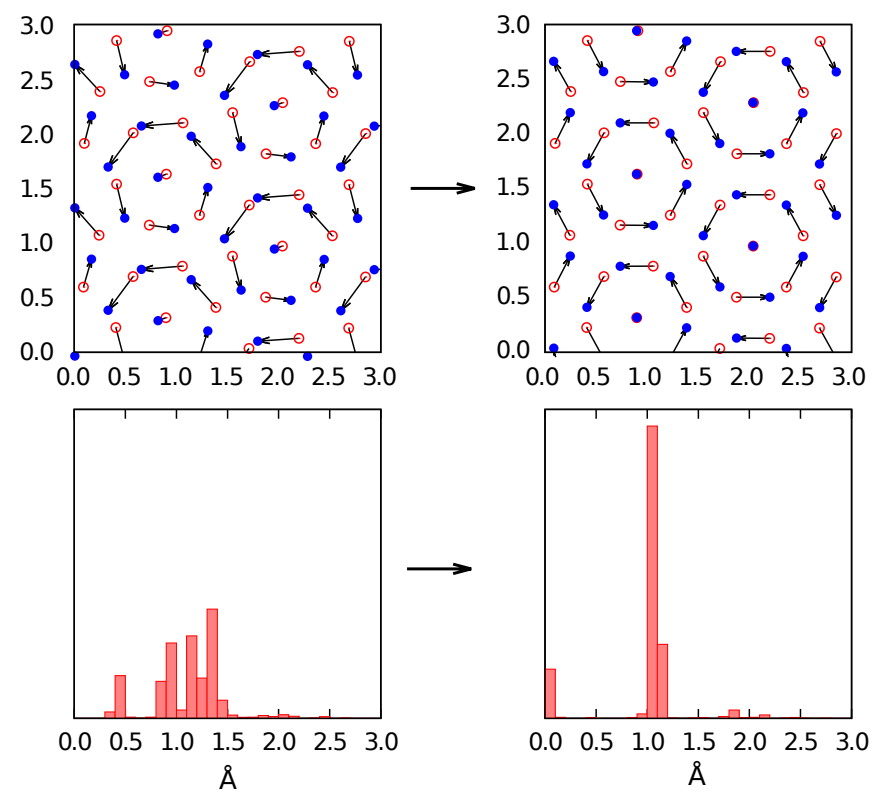

FIG. 7. The atomistic mechanism of grain boundary migration. The top row of figures shows the hops undertaken by atoms as the grain boundary sweeps through from left to right. Open (red) circles indicate the initial positions, filled (blue) circles the final positions. The bottom row of figures shows histograms of the length in $\AA$ of the atomic hops. The figures on the left show the raw data from the migration simulation. The figures on the right are the result of shifting the final positions of the atoms by a fixed translation. Data are for a simulation with $T=400 \mathrm{~K}$ and $P=25 \mathrm{meV} /$ atom $(250 \mathrm{MPa})$.

In our NEB calculations of the collective shuffling of atoms involved in grain boundary migration we constructed two long thin simulation cells, one with a pair of grain boundaries in an initial position and a second with the boundaries in positions displaced by one unit of migration. These are used as the start and end points of NEB calculations using 16 replicas.

To assess the suitability of our chosen EAM empirical potential for the simulation of grain boundary migration we have also carried out similar NEB calculations for the migration barriers of a pair of boundaries in small cells in the DFT code VASP 29. Our supercells were $15.8 \times 13.1 \times 7.0 \AA^{3}$, contained 84 atoms and were periodic in all three dimensions (containing a pair of equivalent grain boundaries). The calculations used the projector augmented wave method [30] and a generalized gradient approximation to the exchange correlation functional 31. A gamma-centred Monkhorst-Pack $3 \times 3 \times 6 \mathrm{k}$-point mesh 32] was used to sample the Brillouin zone and a plane wave cut-off of $380 \mathrm{eV}$ was applied.

Fig. 8 compares the atomic trajectories obtained from NEB calculations with those in a small volume of crystal taken from a much larger dynamic simulation. We see that the NEB algorithm has successfully captured the three-stage migration process evident in the dynamic data. This three stage migration process is further elu- 
cidated in Fig. 9, which compares the evolution of the positions of individual atoms between their initial and final positions with the overall reaction coordinate (in the case of the NEB calculation) or elapsed time (in the case of the simulation). We can see that the emergent mechanism in the dynamic simulation also involves three stages. Of course, being a thermally activated process, the random hopping of the pairs of atoms in the dynamic simulations is not perfectly simultaneous.

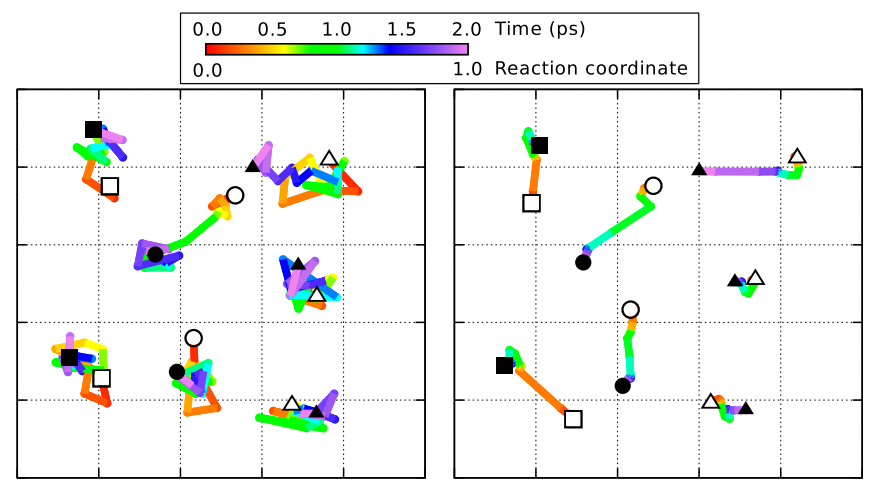

FIG. 8. The migration paths of a group of seven nonequivalent atoms in the CSL cell (left) during a dynamical simulation and (right) as predicted by the NEB calculation. The trajectories are coloured (from red, through green, to blue) according to time from initial (open symbols) to fully migrated positions (closed symbols). In the case of the NEB calculations, the reaction coordinate stands as a proxy for time.

\section{REFERENCES}

[1] C. Deng and C.A. Schuh. Atomistic simulation of slow grain boundary motion. Physical Review Letters, 106(4):45503, 2011.

[2] Stephen M. Foiles and J. J. Hoyt. Computation of grain boundary stiffness and mobility from boundary fluctuations. Acta Materialia, 54(12):3351-3357, 72006.

[3] Rasmus B. Godiksen, Zachary T. Trautt, Moneesh Upmanyu, Jakob Schitz, Dorte Juul Jensen, and Sren Schmidt. Simulations of boundary migration during recrystallization using molecular dynamics. Acta Materialia, 55(18):6383 - 6391, 2007.

[4] Koenraad G. F. Janssens, David Olmsted, Elizabeth A. Holm, Stephen M. Foiles, Steven J. Plimpton, and Peter M. Derlet. Computing the mobility of grain boundaries. Nat Mater, 5(2):124-127, 022006.

[5] David L. Olmsted, Elizabeth A. Holm, and Stephen M. Foiles. Survey of computed grain boundary properties in face-centered cubic metals-ii: Grain boundary mobility. Acta Materialia, 57(13):3704 - 3713, 2009.

[6] B. Schonfelder, G. Gottstein, and L. Shvindlerman. Atomistic simulations of grain boundary migration in copper. Metallurgical and Materials Transactions A, 37:1757-1771(15), June 2006.

[7] B. Schönfelder, D. Wolf, S. R. Phillpot, and M. Furtkamp. Molecular-dynamics method for the simulation of grain-boundary migration. Interface Science,
5(4):245-262, 111997.

[8] I. Toda-Caraballo, P. D. Bristowe, and C. Capdevila. A molecular dynamics study of grain boundary free energies, migration mechanisms and mobilities in a bcc fe20cr alloy. Acta Materialia, 60(3):1116-1128, 22012.

[9] Zachary T. Trautt, Moneesh Upmanyu, and Alain Karma. Interface mobility from interface random walk. Science, 314(5799):632-635, 102006.

[10] M. Upmanyu, R. W. Smith, and D. J. Srolovitz. Atomistic simulation of curvature driven grain boundary migration. Interface Science, 6(1):41-58, 1998-02-01.

[11] H. Zhang, M. I. Mendelev, and D. J. Srolovitz. Computer simulation of the elastically driven migration of a flat grain boundary. Acta Materialia, 52(9):2569-2576, 5 2004 .

[12] H. Zhang, M. Upmanyu, and D.J. Srolovitz. Curvature driven grain boundary migration in aluminum: molecular dynamics simulations. Acta Materialia, 53(1):79 - 86, 2005.

[13] Hao Zhang, Mikhail I. Mendelev, and David J. Srolovitz. Mobility of [sigma] 5 tilt grain boundaries: Inclination dependence. Scripta Materialia, 52(12):1193 - 1198, 2005.

[14] G. Gottstein and L.S. Shvindlerman. Grain Boundary Migration in Metals - Thermodynamics, Kinetics, Applications. CRC Press, 2010.

[15] C. P. Race, J. von Pezold, and J. Neugebauer. Role of the 

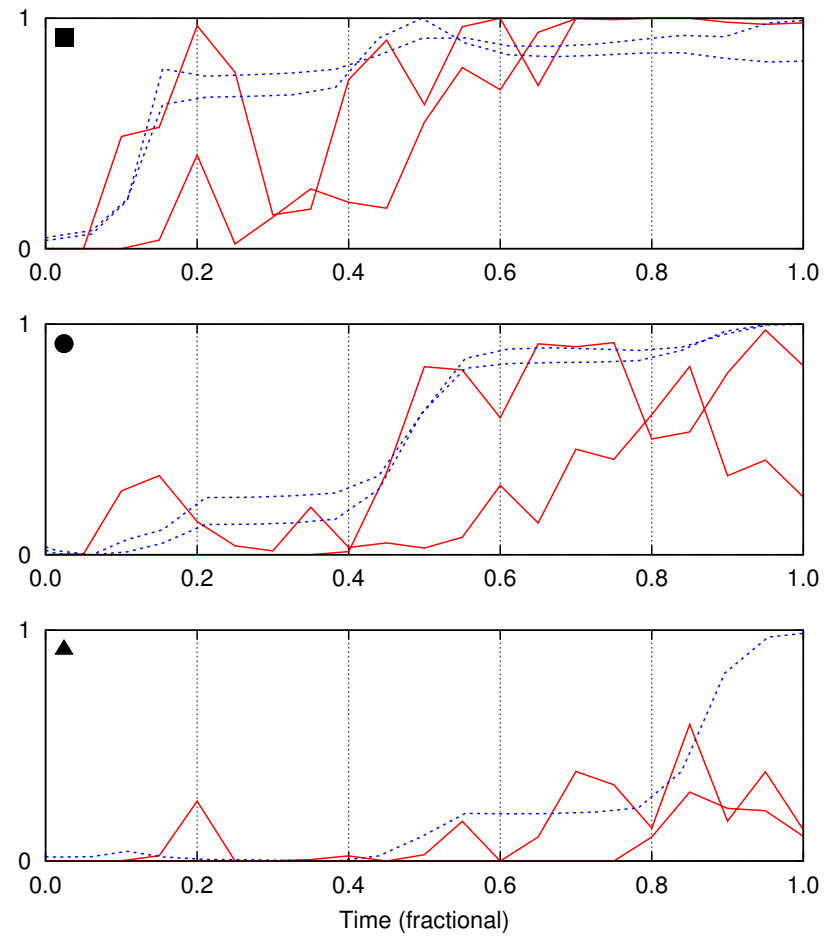

FIG. 9. The reaction coordinate as a function of time for individual atoms in the minimal groups identified by differently shaped symbols in Fig. 8 Data from the dynamical simulations (solid (red) lines) are compared with the predictions of the NEB calculations (dashed (blue) lines), for which the overall reaction coordinate of the NEB cell is used as a proxy for time.

mesoscale in migration kinetics of flat grain boundaries. Physical Review B, 89(21):214110, June 2014.

[16] David L. Olmsted, Stephen M. Foiles, and Elizabeth A. Holm. Grain boundary interface roughening transition and its effect on grain boundary mobility for non-faceting boundaries. Scripta Materialia, 57(12):1161-1164, 12 2007.

[17] A. P. Sutton and R. W. Balluffi. Interfaces in crystalline materials. Oxford: Clarendon Press, 1995.

[18] A. P. Sutton and V. Vitek. On the structure of tilt grain boundaries in cubic metals i. symmetrical tilt boundaries. Philosophical Transactions of the Royal Society of
London. Series A, Mathematical and Physical Sciences, 309(1506):1-36, 1983.

[19] Rajendra R. Zope and Y. Mishin. Interatomic potentials for atomistic simulations of the ti-al system. Phys. Rev. B, 68(2):024102, Jul 2003.

[20] C.P. Race. Quantifying uncertainty in molecular dynamics simulations of grain boundary migration. Molecular Simulation, 0(0):1-5, January 2014.

[21] H. Song and J. J. Hoyt. An atomistic simulation study of the migration of an austenite-ferrite interface in pure fe. Acta Materialia, 61(4):1189-1196, 22013.

[22] R. Würschum and R. W. Balluffi. In-situ study of the migration of grain boundary facets in au bicrystals under high driving forces. physica status solidi (a), 136(2):323336, 1993.

[23] Hao Zhang and David J. Srolovitz. Simulation and analysis of the migration mechanism of [sigma] 5 tilt grain boundaries in an fcc metal. Acta Materialia, 54(3):623633, 22006.

[24] Graeme Henkelman, Blas P. Uberuaga, and Hannes Jonsson. A climbing image nudged elastic band method for finding saddle points and minimum energy paths. The Journal of Chemical Physics, 113(22):9901-9904, 2000.

[25] Suk-Joong L. Kang, Min-Gon Lee, and Se-Min An. Microstructural evolution during sintering with control of the interface structure. Journal of the American Ceramic Society, 92(7):1464-1471, 2009.

[26] For comparison with the dynamical simulations this barrier was calculated using the NEB method in a cell in which the lattice parameter had been enlarged to the value for our chosen empirical potential at $600 \mathrm{~K}$.

[27] Steve Plimpton. Fast parallel algorithms for short-range molecular dynamics. Journal of Computational Physics, 117(1):1-19, 31995.

[28] Graeme Henkelman and Hannes Jonsson. Improved tangent estimate in the nudged elastic band method for finding minimum energy paths and saddle points. The Journal of Chemical Physics, 113(22):9978-9985, 2000.

[29] G. Kresse and J. Hafner. Ab initio molecular dynamics for liquid metals. Phys. Rev. B, 47:558, 1993.

[30] P. E. Blöchl. Projector augmented-wave method. Phys. Rev. B, 50:17953, 1994.

[31] J. P. Perdew, K. Burke, and M. Ernzerhof. Generalized gradient approximation made simple. Phys. Rev. Lett., 77:3865, 1996.

[32] Hendrik J. Monkhorst and James D. Pack. Special points for brillouin-zone integrations. Phys. Rev. B, 13:51885192, Jun 1976. 